\title{
Switchable wiring for high-resolution electronic measurements at very low temperatures
}

\author{
C. Schirm, H.-F. Pernau, and E. Scheer \\ Department of Physics, University of Konstanz, D-78457 Konstanz, Germany
}

(Received 2 November 2008; accepted 1 January 2009; published online 19 February 2009)

\begin{abstract}
Low-temperature transport measurements with high energy resolution require effective filtering of high-frequency input. The high dc resistance of standard $R C$ filters results in considerable heat input and hampers measurements with high currents or voltages. We developed a wiring scheme that incorporates a commercial latching relay at very low temperature between two sets of wires. In our application one set of wires comprises a voltage divider and a high-Ohmic reference resistance at low temperature as well. The other set has low dc resistance and no voltage divider. Both sets are high frequency filtered with very robust and compact filters, though, for insuring effective damping at gigahertz frequencies. We demonstrate that with the first set, we obtain a voltage resolution of $6 \mu \mathrm{V}$ and a current resolution of $100 \mathrm{pA}$, which is sufficient for the recording and analysis of multiparticle transport in superconducting point contacts. The second set is used for electromigration experiments on superconducting point contacts and allows application of currents up to $1 \mathrm{~mA}$ and voltages up to $20 \mathrm{~V}$, while the sample is at $1 \mathrm{~K}$. More versatile applications of the scheme are possible.
\end{abstract}

\section{INTRODUCTION}

Many interesting quantum transport properties do occur at very low temperatures below $1 \mathrm{~K}$ only. Examples are experiments on solid-state quantum computing devices ${ }^{1}$ or tunnel spectroscopy. ${ }^{2}$ In the millikelvin range thermalization of the sample and the electronic system within the sample becomes a difficult task because of the strong temperature dependence of Planck's radiation law. In order to guarantee that-in spite of the unavoidable connections to the room temperature apparatus - the measurement is performed at thermal equilibrium, the whole cryostat can be mounted into a high-frequency shielded room. ${ }^{3}$ This is however cost intensive, space consuming, and difficult to operate. As an alternative the electric lines are usually thoroughly filtered in the radio-frequency range and above. The easiest way is to use $R C$ filters consisting of standard discrete electronic elements. However, obtaining sufficient damping at high frequency usually results in considerable $R$ values and thus strong dissipation of limiting the base temperature of the cryostat. Furthermore the usual filter characteristic is rather shallow, i.e., giving rise to signal deterioration at low frequency as well. This problem can be solved by using copper-powder ${ }^{4}$ or microfabricated thin-film filter elements ${ }^{5}$ and/or coaxial wires. ${ }^{6,7}$ While copper-powder filters are bulky, the thin-film elements do in general hinder the application of high currents $(>10 \mu \mathrm{A})$ or high voltages $(>1 \mathrm{~V})$. It is therefore helpful to have the possibility to switch between two or more sets of electric wirings with different performance without loss of energy resolution, while the sample is kept at low temperatures. We describe a setup in which a relay switch mounted at $250 \mathrm{mK}$ is used for alternating between wiring designed for spectroscopic measurements on a superconducting atomic contact and a high-current wiring used for electromi- gration experiments on the same sample while it is kept cold. The switching principle can, however, be applied to more versatile cabling as well.

\section{EXPERIMENTAL}

The cryostat is a commercial ${ }^{3} \mathrm{He}$ bath from Oxford Instruments (type Heliox-VT) with a cooling power of approximately $40 \mu \mathrm{W}$ at $300 \mathrm{mK}$. Due to the limited cooling power of the so-called $1 \mathrm{~K}$ stage, the number and heat input of the electric lines have to be reduced to the minimum in order to arrive at the base temperature of $250 \mathrm{mK}$ for a reasonable time span of $24 \mathrm{~h}$. The design of the cryostat is optimized to hasten cool down and warm up and provides thus limited possibility for the thermalization of the wiring. We had best results when using continuous, home-made wires without interruption down to the ${ }^{3} \mathrm{He}$ pot.

Figure 1 shows the wiring which we used in a standard ${ }^{3} \mathrm{He}$ cryostat. The sets of wires labeled $U_{+} / U_{-}, I_{+} / I_{-}$, and $B_{+} / B_{-}$are three pairs of twisted Manganin ${ }^{\circledR}$ wires [diameter of $50 \mu \mathrm{m}, 224.9 \Omega / \mathrm{m}$, twofold Kapton $\AA^{8}$ electrical isolation, type Ed 2L-W 200 (Ref. 9)] in a stainless steel tube (outer diameter of $0.4 \mathrm{~mm}$ and inner diameter $0.2 \mathrm{~mm}$ ). As electrolyte we use silicone dissolved in toluene for matching the capacitance. These wires were adapted from Refs. 6 and 7. In our cryostat they have a total length of $1.5 \mathrm{~m}$. The capacitance of such a wire is about $250 \mathrm{pF} / \mathrm{m}$. The cable labeled $U_{R}$ is a coaxial wire made of brass with a diameter of $112 \mu \mathrm{m}$ (Ref. 10) in an equivalent stainless steel tube and filled with silicone-toluene mixture as well, resulting in a resistance of $15.4 \Omega$ and a capacitance of $430 \mathrm{pF} / \mathrm{m}$. The wires thus serve as low-pass filters with an effective calculated cutting frequency of $14 \mathrm{MHz}$ (twisted pairs) or 186 $\mathrm{MHz}$ (coaxial wire), respectively, and provide high- 


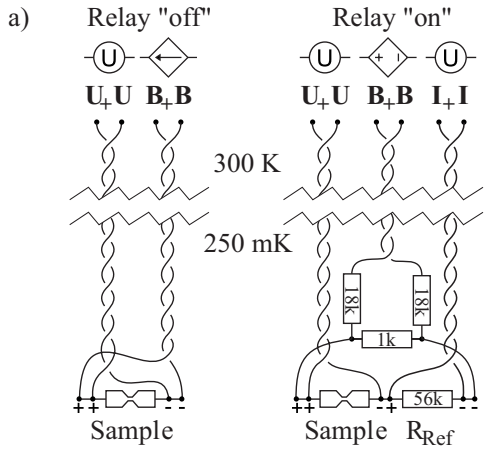

b)

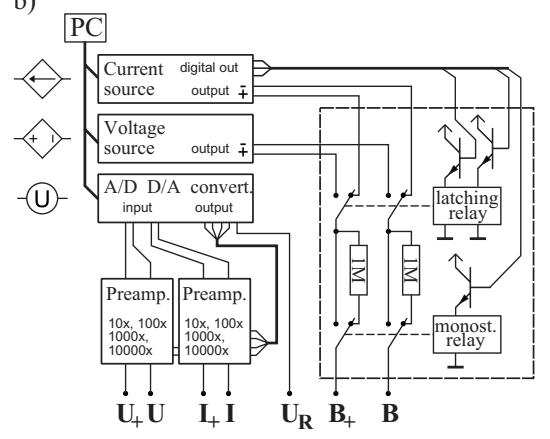

c)

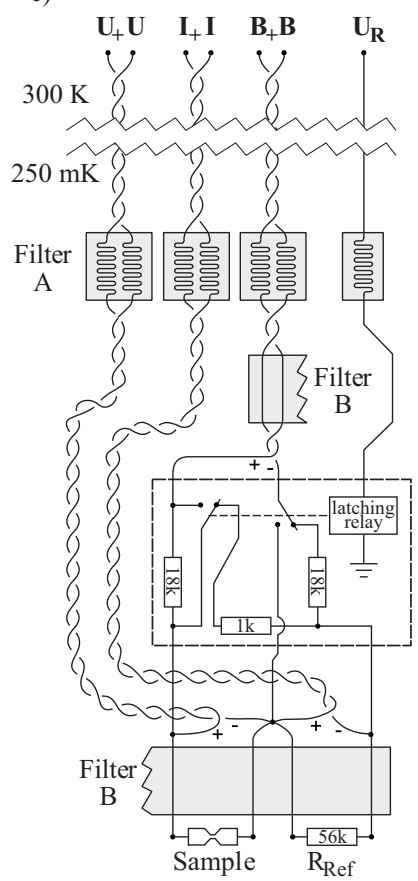

FIG. 1. (a) Effective wiring in "off" (left) and "on" (right) switch positions. (b) Scheme of the wiring and devices outside the cryostat. (c) Scheme of the wiring inside the cryostat. Complete wiring also showing the discrete filter boxes. frequency damping of -58 or $-64 \mathrm{~dB}$ at $5 \mathrm{GHz}$. The tubes are squeezed between copper mesh, which are screwed to the 4.2 and $1 \mathrm{~K}$ flange of the cryostat. Besides this $R C$ filtering all cables connecting the sample are additionally equipped with two types of home-made copper-powder filters to reduce the electronic temperature. The upper filters [type A, dc resistance of $16 \Omega$, see Fig. 2(a)] are small brass boxes and are thermally anchored via a brass screw to the ${ }^{3} \mathrm{He}$ pot. They contain $2.4 \mathrm{~m}$ Kapton-shielded copper wires in each line [diameter of $50 \mu \mathrm{m}$ (Ref. 11)]. The wires are wound in mutually inverted loops, as shown in Fig. 2(b), around four posts in order to limit the inductance to less than $3 \mu \mathrm{H}$. One pair of wires is fed through one filter box each. The grain size of the copper powder is $<40 \mu \mathrm{m}$. These filters provide a damping of $-70 \mathrm{~dB}$ at $600 \mathrm{MHz}$. The lower filter box (type $\mathrm{B}$, made of copper, outer dimension of $21 \times 24 \times 4 \mathrm{~mm}^{3}$ for all seven channels) in ultimate proximity to the sample contains for each channel a very short $(5 \mathrm{~cm})$ wire of nonisolated copper wire and is shown to be crucial for obtaining

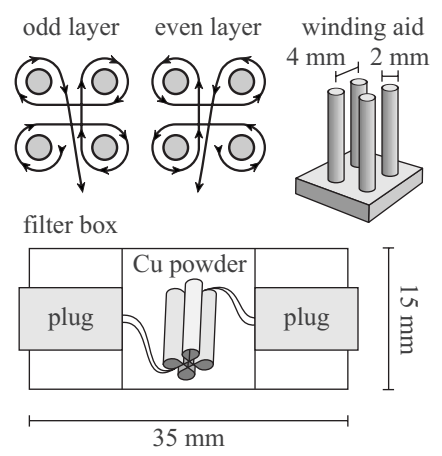

FIG. 2. Filter design and fabrication scheme. Top left: Mutually inverted winding scheme for reducing the inductance. Top right: Tool used for performing the winding. Bottom left: Top view of type A filters. For single line filters, the plugs are SMA plugs; for twisted pairs, they are Fischer type S101 plugs (Ref. 12). the required energy resolution for the spectroscopic measurements. The damping per wire length of these filters with a cutoff frequency around $2 \mathrm{GHz}$ and a damping of $\sim-20 \mathrm{~dB}$ is higher than that of type A filters because of the better coupling of the nonisolated wire to the copper powder. Besides damping it performs the task of cooling of the sample by heat conduction through the wire, in particular, to reduce the heat input from the relay and the voltage divider. The bias lines $\left(B_{+}, B_{-}\right)$contain an additional type A copper-powder filter and a 37:1 voltage divider for reducing the noise input from the voltage source. The voltage divider is built up in a symmetric way from three metal-film resistors with 18,1 , and $18 \mathrm{k} \Omega$ and is also installed in a small copper box thermally connected to the ${ }^{3} \mathrm{He}$ pot. Although the nominal resolution of the current source should be sufficient to use it also for the low-current measurements without the voltage divider, it is difficult to achieve in a real circuit including low-temperature wiring. Without the voltage divider the actual sample resistance determines the relative voltage drops across the individual elements of the circuit and the position of the virtual ground level. The addition of a voltage divider fixes the majority of the voltage drop at this voltage divider and thus allows us to optimize the circuit according to this working point. The main improvement of our setup is that we rethermalize the wires after the heat dissipation through the voltage divider by the type B filter. The electrical connections within the cryostat are provided by SubMiniature-A (SMA) connectors (coaxial wire) or triaxial connectors (twisted-pair wires) ${ }^{12}$ or by soldering with a lowmelting temperature soldering alloy. ${ }^{13}$

A latching relay ${ }^{14}$ mounted at $T=250 \mathrm{mK}$ switches between the two wirings. It is a two-coil bistable relay, which requires a voltage signal of $5 \mathrm{~V}$ for operation. In principle it would be possible to switch both coils independently by two control lines. In order to reduce the total number of connec- 


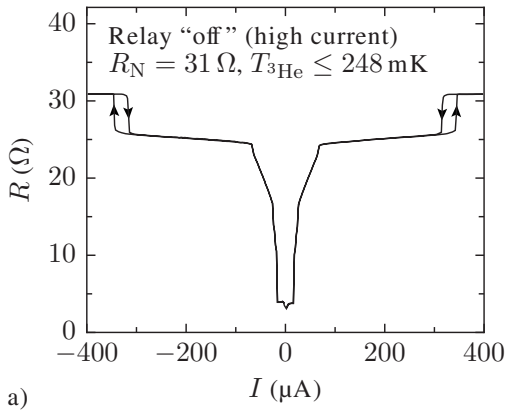

tions and thus the heat load from room temperature to the base temperature, we decided to use only one coil and to use both polarities of $U_{R}$ instead. In our setup it is connected in such a way that it switches "on" and "off" the voltage divider and the reference resistance of $56 \mathrm{k} \Omega$ close to the sample, which we use for determining the current in the spectroscopic measurements. In the off state the wiring represents a standard four-wire resistance measurement with the $B$ lines serving for applying the current and the $U$ lines for measuring the voltage across the sample. The $I$ lines are out of use. In the on state the $B$ lines provide a voltage bias across the series of the sample and the reference resistance $R_{\text {ref }}$; with the $I$ lines and the $U$ lines we measure the current and the voltage through and across the sample.

\section{RESULTS}

\section{A. Function and resolution of the setup}

In atomic-size contacts the electrical conductance is determined by the exact position and configuration of each single atom and by the local environment around the contact. $^{15}$ The transport is described by the Landauer theory, which treats it as a quantum mechanical scattering problem. The conductance of a contact is carried by a small number of conduction channels. The number and transmission coefficients of these channels are determined by the chemical valence and the atomic configuration. ${ }^{16}$ The usual methods for establishing such contacts do not allow us to define and to measure the positions of all relevant atoms with atomic precision and to perform modifications on the level of individual atoms.

To fabricate and investigate a large number of different atomic contacts, we use lithographically defined mechanically controllable break junctions $(\mathrm{MCBs})^{15,17}$ made of the superconducting element aluminum. The adjustment is performed by uniformly bending the substrate. A pushing rod is placed at the rear side of the device close to the break junction and two countersupports ensure the bending. The contacts are formed randomly after every closing and opening cycle.

Figures 3(a) and 3(b) display measurements of a superconducting $\mathrm{Al}$ sample in two different geometrical configurations measured with both wirings when the thermometer at the ${ }^{3} \mathrm{He}$ pot read temperatures $T_{3^{3} \mathrm{He}}$ below $250 \mathrm{mK}$. Figure 3(b) shows a current-voltage characteristic $(I V)$ of an atomicsize $\mathrm{Al}$ contact arranged via a MCB with the switch in the on position, recorded in a voltage range of $-40-40 \mu \mathrm{V}$. The inset shows the same trace in a wider voltage range of
$-450-450 \mu \mathrm{V}$. In this resistance range the contact represents a Josephson contact with a finite supercurrent, which can be described by the resistively and capacitively shunted Josephson contact model in the underdamped regime. ${ }^{18}$ Above a voltage corresponding to the superconducting gap of $\mathrm{Al}$ of $2 \Delta=360 \mu \mathrm{eV}, I V$ is linear; below, it displays nonlinearities due to multiple Andreev reflections (MARs), ${ }^{19}$ which will be further discussed below. We here concentrate on the supercurrent branch in order to demonstrate the voltage and current resolution of our wirings. A finite slope of the supercurrent branch is observed, which could be caused by phase diffusion. When neglecting phase diffusion effects, the finite slope could also be given by finite series resistances of the circuit, which we have measured independently to be less than $0.1 \Omega$. The third possible origin of the finite slope would thus be the finite voltage resolution of the setup and it can thus be used to determine an upper bound for this important quantity. The voltage resolution denotes the precision with which a voltage of a given value can be applied. This property differs from the voltage noise, which denotes the precision with which a voltage value can be detected. The latter one can be reduced by longer measuring times and repeated measurements and averaging, while the first one is given by the quality of the electronic wiring, the highfrequency input, and the thermalization. The traces in Fig. 3 have been recorded without averaging with a measuring time of $0.1 \mathrm{~s} /$ point. An upper bound for the voltage resolution is surely given by the width of the supercurrent branch. As can be seen from Fig. 3(b), it is better than $6 \mu \mathrm{V}$, while the voltage noise, i.e., the scattering from point to point, is less than $1 \mu \mathrm{V}$ (in the given example). Equivalently we determine the quantities current resolution, which is better than $100 \mathrm{pA}$, and current noise, which we estimate to less than $10 \mathrm{pA}$.

However, the high Ohmic resistance of this wiring including the voltage divider hinders the application of currents larger than approximately $10 \mu \mathrm{A}$ through the sample. Higher bias signals result in strong heating of the whole cryostat inset (detected as the increase in $T^{3} \mathrm{He}$ ) or destroy the electric lines or filters. This problem is solved when switching the relay to the off state [Fig. 3(a)]. Figure 3(a) shows the breakdown of the supercurrent at the closed break junction with the switch in the off position upon application of a high bias current. The first resistance step corresponds to the critical current value of the narrowest part of the break junction, which is about $100 \times 100 \mathrm{~nm}^{2}$. The further increase reflects the widening of the leads of the break junction until finally 


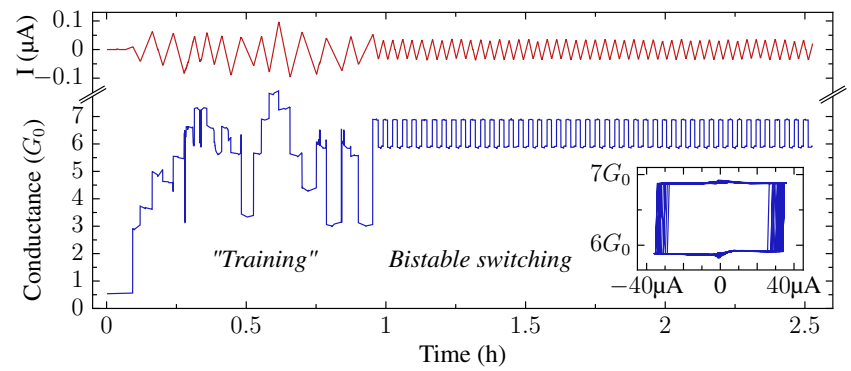

FIG. 4. (Color online) Creation of a bistable atomic switch. Upper trace: Control current applied for creating the rearrangements as a function of time. Lower trace: Conductance of a MCB structure made from $\mathrm{Al}$ as a function of time when applying the control current given above. Inset: Plot of conductance vs control current for the bistable part.

around $320 \mu \mathrm{A}$ the critical current of the $\mathrm{Al}$ film is overcome. This latter resistance step is slightly hysteretic. The width of the hysteresis corresponds to a temperature increase in the sample induced by the bias current of about $30 \mathrm{mK}$. Thus, in spite of the high current the additional thermal load is small enough for the ${ }^{3} \mathrm{He}$ system to stay below $T^{{ }^{3}} \mathrm{He}$ $<250 \mathrm{mK}$, while the sample temperature stays roughly below $300 \mathrm{mK}$. Both would be impossible with the voltage divider in the on position. The voltage resolution of the wiring in the off position is estimated to be about $100 \mu \mathrm{V}$ and this wiring is thus unsuitable for measuring effects at voltage scales below $50 \mu \mathrm{V}$, as the ones shown in Fig. 3(b).

\section{B. Electromigration of atomic-size contacts}

As a second way to influence the atomic structure, we induce local electromigration at the contact by applying high electrical currents. In particular situations a reversible switching between two conductance values is observed, which is similar to that obtained earlier by an electrochemical gating technique. ${ }^{20}$

Electromigration experiments can be performed using the low resistive cables, i.e., with the switch in the off position. Figure 4 depicts a special type of experiment, which may lead with good yield to a configuration in which the conductance can be switched reversibly between two values (>500 times). This can be done by alternating current ramps that change their direction on every detected jump in the conductance. The initial conductance is adjusted by the MCB setup. After a "training" period with alternating ramps, a bistable situation can emerge. The inset of Fig. 4 shows the hysteresis loop of such a situation as a function of the applied current.

An interesting set of questions is open in the field of electromigration at the atomic level. ${ }^{21}$ Single-atomic electromigration events can be studied in our experiment. One still open question is the origin of the maxima in the conductance histograms of multivalent metals. ${ }^{15,22}$ In monovalent metals such as gold, the preferred conductance values coincide with a saturation of the transmission of the channels. ${ }^{15}$ This does not hold for multivalent metals such as aluminum for which in the single-atom contact three channels with arbitrary transmission add up in such a way that the total conductance has a preferred value. ${ }^{22,23}$ Several mechanisms including electronic correlation effects ${ }^{24}$ or structural effects ${ }^{25}$ have been suggested. With the help of atomic-level electromigration, we are able to study which conductance values belong to particularly stable atomic configurations. By combination of electromigration and high-resolution $I V$ characteristic measurements in the superconducting state, we deduce which quantum mechanical modes they carry. ${ }^{16,23}$ An example of such a combined study is shown in Fig. 5. In the top of the right panel, we plot as in Fig. 4 the conductance as a function of time while ramping the current. A repeated switching between two values is observed. In addition we plot the transmission coefficients deduced from analyzing the superconducting $I V$ 's at low voltage. The dotted curves in the left panel show two examples of $I V$ characteristics recorded on the upper and the lower levels of the right panel. The black lines are with fits to the theory of MAR from which the channels can be determined unambiguously. In the given examples the $I V$ 's can be decomposed into two channels in the lower level and three channels in the upper level, with the transmission coefficients given in the figure caption. As can be read from the right panel, the channel content is the same in all repetitions. This proves that we can switch between exactly the same two atomic configurations. In the given example all channels have noninteger transmission coefficients and all are affected by the reconfiguration. In the upper level with three channels, two transmission coefficients are very close to each other. The appearance of two channels with almost equal transmission suggests a highly symmetric configuration. Our findings are in reasonable agreement with a calculation for a single-atom contact between two perfectly
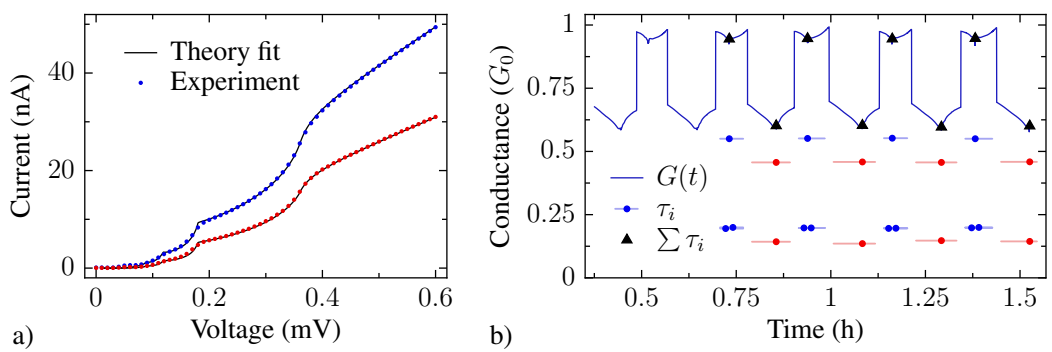

FIG. 5. (Color online) Left panel: Current-voltage characteristics of two atomic contacts of an Al sample measured at $280 \mathrm{mK}$ and fits to the theory of MAR (Ref. 12) from which we deduce the conduction channels with transmissions $\tau_{1}=0.195, \tau_{2}=0.200$, and $\tau_{3}=0.550$ (upper curve, blue online) and $\tau_{1}=0.143$ und $\tau_{2}=0.457$ (lower curve, red online). Right panel: Conductance vs time of an Al MCB and decomposition into channels for the bistable situation from which two examples of $I V$ 's are shown in the left panel. The black triangles indicate the sum of $\tau_{i}$ and correspond well to the conductance measured with the wiring in the "off" position (straight line). 
ordered pyramids in the (111) direction, for which two degenerate channels with transmissions close to 0.15 and a dominating channel with a transmission of about 0.8 have been found. ${ }^{26}$ A two-channel configuration, as we find for the lower conductance value, could either be provided by a socalled dimer contact, i.e., a two-atom long chain, or a disordered single-atom contact. ${ }^{27}$ A detailed analysis of bistable atomic configurations will be published elsewhere.

\section{CONCLUSIONS}

In conclusion, we have presented a cryostat wiring that combines high-frequency damping necessary for spectroscopic measurements with the possibility to apply large transport currents as required, e.g., for electromigration experiments on atomic contacts. We demonstrated the resolution and current bearing performance with both wirings. The technique overcomes the limitation of existing standard cryostat wirings by the application of a latching relay at very low temperature, which alternates between two different wiring schemes. The presence of effective high-frequency filters in both wirings is necessary for achieving the required energy resolution of approximately $6 \mu \mathrm{eV}$.

\section{ACKNOWLEDGMENTS}

We are indebted to C. Debuschewitz, T. Geiger, V. Kunej, F. Otto, H. Richter, O. Schecker, C. Strunk, and M. Wolz for experimental assistance and discussions about cryogenics issues. We have enjoyed fruitful discussions with J. C. Cuevas and M. Häfner. We gratefully acknowledge financial support from the DFG through SFB513.

${ }^{1}$ D. Vion, A. Aassime, A. Cottet, P. Joyez, H. Pothier, C. Urbina, D. Esteve, and M. H. Devoret, Science 296, 886 (2002).

${ }^{2}$ D. C. Ralph, C. T. Black, and M. Tinkham, Phys. Rev. Lett. 78, 4087 (1997).

${ }^{3}$ A. Wallraff, A. Lukashenko, C. Coqui, A. Kemp, T. Duty, and A. V. Ustinov, Rev. Sci. Instrum. 74, 3740 (2003).

${ }^{4}$ J. M. Martinis, M. H. Devoret, and J. Clarke, Phys. Rev. B 35, 4682 (1987).
${ }^{5}$ H. Le Sueur and P. Joyez, Rev. Sci. Instrum. 77, 115102 (2006).

${ }^{6}$ D. C. Glattli, P. Jacques, A. Kumar, P. Pari, and L. Saminadayar, J. Appl. Phys. 81, 7350 (1997).

${ }^{7}$ A. B. Zorin, Rev. Sci. Instrum. 66, 4296 (1995).

${ }^{8}$ Kapton ${ }^{\circledR}$ is a registered trademark of DuPont, DuPont de Nemours (Luxembourg) s.a r.1., Rue General Patton, L-2984 Luxembourg.

${ }^{9}$ Manganin ${ }^{\circledR}$ is a registered trademark from Isabellenhütte Heusler $\mathrm{GmbH}$, D-35664 Dillenburg.

${ }^{10}$ Purchased from GVL Cryoengineering, Aachener Str. 89, D-52223 Stolberg.

${ }^{11}$ Purchased from Detakta Hans-Böckler-Ring 19, D-22851 Norderstedt.

${ }^{12}$ Fischer Connectors SA type S101A005-120 Chemin du Glapin 20,CH1196 Saint-Prex.

${ }^{13}$ Compositions of $43 \% \mathrm{Sn}$ and $57 \%$ Bi purchased from Castolin Eutectics (Castolin $\mathrm{GmbH}$, Gutenbergstraße 10, D-65830 Kriftel). The melting temperature is $T_{m}=140{ }^{\circ} \mathrm{C}$; the superconducting transition temperature is $T_{c}$ $=2.3 \mathrm{~K}$. The reduction in $T_{c}$ as compared to a standard soldering material has considerably improved the thermalization of the inner wires. We used $\mathrm{ZnCl}$ fluent for enhancing the adhesion to the metal wire.

${ }^{14}$ Type RAL-D 5 W-K, manufacturer Takamisawa, reseller, e.g., Conrad Elektronik, Klaus-Conrad-Str. 1, D-92240 Hirschau, Germany.

${ }^{15}$ N. Agraït, A. Levy Yeyati, and J. M. van Ruitenbeek, Phys. Rep. 377, 81 (2003).

${ }^{16}$ E. Scheer, N. Agraï, J. C. Cuevas, A. Levy Yeyati, B. Ludoph, A. MartínRodero, G. Rubio Bollinger, J. M. van Ruitenbeek, and C. Urbina, Nature (London) 394, 154 (1998).

${ }^{17}$ J. M. van Ruitenbeek, A. Alvarez, I. Piñeyro, C. Grahmann, P. Joyez, M. H. Devoret, D. Esteve, and C. Urbina, Rev. Sci. Instrum. 67, 108 (1996).

${ }^{18}$ M. Tinkham, Introduction to Superconductivity (McGraw-Hill, New York, 1996), Chap. 6.

${ }^{19}$ J. C. Cuevas, A. Martín-Rodero, and A. Levy Yeyati, Phys. Rev. B 54, 7366 (1996).

${ }^{20}$ F. Xie, L. Nittler, Ch. Obermair, and Th. Schimmel, Phys. Rev. Lett. 93, 128303 (2004).

${ }^{21}$ P. Lodder, Europhys. Lett. 72, 774 (2005).

${ }^{22}$ I. A. Yanson and J. M. van Ruitenbeek, Phys. Rev. Lett. 79, 2157 (1997).

${ }^{23}$ E. Scheer, P. Joyez, D. Esteve, C. Urbina, and M. H. Devoret, Phys. Rev. Lett. 78, 3535 (1997).

${ }^{24}$ S. Kirchner, J. Kroha, and E. Scheer, in Proceedings of the NATO Advanced Research Workshop, NATO Science Series II, Pecs, Hungary, 28 May-1 June 2000, edited by V. Chandrasekhar, C. v. Haesendonck, and A. Zawadowski (Kluwer Academic, Dordrecht, Boston, London, 2001), Vol. 50 , p. 215.

${ }^{25}$ I. K. Yanson, O. I. Shklyarevskii, J. M. van Ruitenbeek, and S. Speller, Phys. Rev. B 77, 033411 (2008).

${ }^{26}$ J. C. Cuevas, A. Levy Yeyati, and A. Martín-Rodero, Phys. Rev. Lett. 80, 1066 (1998).

${ }^{27}$ M. Häfner and J. C. Cuevas (private communication). 